\title{
CALCULATIONS ON THE SUPREMUM OF FUZZY NUMBERS VIA $L_{p}$ METRICS
}

\author{
D. WEN AND T. FAN
}

\begin{abstract}
In this paper, it is proved that the supremum of a family of fuzzy numbers can be finitely approximated via $L_{p}$ metrics and the concrete approaches are given. As a byproduct, it is proved that the $L_{1}$ metric $d_{1}$ defined via cut-set is equivalent to a metric which can be calculated directly via membership functions. Since the $L_{p}$ metrics are analytic in nature, the results in this paper may have interesting applications in fuzzy analysis. For example, it may provide a method for the computation of various fuzzy-number-valued integrals.
\end{abstract}

\section{INTRODUCTION AND PRELIMINARIES}

Since the concept of fuzzy number was first introduced in the 1970's, it has been studied extensively from many different viewpoints. Fuzzy numbers has been used as a basic tool in different parts of fuzzy theory. In [4], it is shown that the endograph metric is approximative with respect to orders on $E^{1}$ and it is computable. From [3], we know that for uniformly supported bounded set of fuzzy numbers the $L_{p}$ metrics and the endograph metric are equivalent. Thus, we can conclude that $L_{p}$ metrics are also approximative. In [2], a method for calculating supremum and infimum of fuzzy sets via endograph metric is given which resembles the Riemann sum in calculus. In this paper, we will give out the concrete methods to approximate the supremum via $L_{p}$ metrics.

First of all, we recall the basics of fuzzy numbers. Let $R$ and $I$ be the set of all real numbers and the unit interval respectively.

Received 28 June 2006, revised 1 November 2006, accepted 13 November 2007. 2000 Mathematics Subject Classification: 54E35, 06F30

Key words and Phrases: Fuzzy numbers, $L_{p}$ metrics, approximation, supremum 
Denote $E^{1}=\{u \mid u: R \rightarrow I, u$ has the following properties $(i)-(i v)\}$.

(i) $u$ is normal, i.e., $u\left(x_{0}\right)=1$ for some $x_{0} \in R$;

(ii) $\mathrm{u}$ is quasiconvex(fuzzy convex), i.e., $u(r x+(1-r) y) \geq \min \{u(x), u(y)\}$ for all $x, y \in R$ and $r \in I$;

(iii) $u$ is upper semicontinuous(u. s. c. for short);

(iv) The topological support $u_{0}$ of $u$ is compact, where $u_{0}=\operatorname{cl}\{x \mid x \in R, u(x)>0\}$.

Elements in $E^{1}$ are called fuzzy numbers. For $\alpha \in I$, let $u_{\alpha}=\{x \in R \mid u(x) \geq$ $\alpha$ denote the $\alpha-$ cut of $u$, then all cuts of $u$ are none-empty closed intervals. For each $\alpha \in I$, let $u_{\alpha}=\left[u_{\alpha}^{-}, u_{\alpha}^{+}\right]$. For any $u, v 1 E^{1}$, define:

$$
d_{p}(u, v)=\left(\int_{0}^{1}\left(\max \left\{\left|u_{\alpha}^{-}-v_{\alpha}^{-}\right|,\left|u_{\alpha}^{+}-v_{\alpha}^{+}\right|\right\}\right)^{p} d \alpha\right)^{\frac{1}{p}},
$$

where $p \geq 1$ is an arbitrary real number. Then $d_{p}$ is a separable but not complete metric on $E^{1}$, called $L_{p}$ metrics. By the definition of $L_{p}$ metrics, we have the following inequalities.

$$
d_{p}(u, v) \leq\left(\int_{0}^{1}\left(\left|u_{\alpha}^{-}-v_{\alpha}^{-}\right|+\left|u_{\alpha}^{+}-v_{\alpha}^{+}\right|\right)^{p} d \alpha\right)^{\frac{1}{p}} \leq\left\|u^{-}-v^{-}\right\|_{p}+\left\|u^{+}-v^{+}\right\|_{p},
$$

where $\|\cdot\|_{p}=\left(\int_{0}^{1}|\cdot|^{p} d \alpha\right)^{\frac{1}{p}}$.

We note that in the literature, fuzzy numbers can also be equivalently defined as follows: $u=\left(l_{u}, r_{u}\right)$, where $l_{u}$ and $r_{u}$ are functions defined on certain closed intervals $\left[a_{u}, c_{u}\right]$ and $\left[c_{u}, b_{u}\right]$ with codomain $I$ respectively, such that $l_{u}\left(a_{u}\right)=0$, $l_{u}\left(c_{u}\right)=1, r_{u}\left(c_{u}\right)=1, r_{u}\left(b_{u}\right)=0$; they are increasing and decreasing respectively, and both are upper $\mathrm{u}$. s. c.. For our need we require that the domains of definition for $l_{u}$ and $r_{u}$ are the whole real line $R$, so that they can be extended uniquely to keep their monotonicity. That is, outside their domains, their values should be either 0 or 1 according to the monotonicity requirement.

For $u, v \in E^{1}$, define $u \leq v$ if only if $u_{\alpha}^{-} \leq v_{\alpha}^{-}, u_{\alpha}^{+} \leq v_{\alpha}^{+}$for all $\alpha \in I$, or equivalently, $l_{u} \geq l_{v}$ and $r_{u} \leq r_{v}$. Then $\leq$ is a partial order on $E^{1}$.

For other undefined notions, we refer to [1].

Remark 1 By the definition of order relation on $E^{1}$ and the definition of $L_{p}$ metrics, it is obvious that for $u, v, w \in E^{1}$, if $u \leq v \leq w$, then $d_{p}(u, v) \leq d_{p}(u, w)$, i.e., $L_{p}$ metrics preserve the order on fuzzy numbers.

\section{CALCULATIONS VIA $L_{1}$ METRIC}

In this section, we give some concrete calculation methods to approximate the supremum of fuzzy numbers via $L_{1}$ metric. Moreover, we prove that the $L_{1}$ metric defined via cut-set can be directly studied by a metric on $E^{1}$ which is defined via membership functions and give out the calculation approach on the supremum directly via membership function method.

Proposition 1[8] Let $\left\{u_{t} \mid t \in T\right\}$ be a family of fuzzy numbers with $\alpha$ - cut representations $\left\{\left(u_{t}\right)_{\alpha} \mid \alpha \in[0,1]\right\}, t \in T$. If the family is bounded above, and $v$ is the 
supremum of the family, then the cut-set functions of $v$ have the following representation:

$$
\begin{aligned}
& v_{\alpha}^{-}=\bigvee_{t \in T}\left(u_{t}\right)_{\alpha}^{-}, \\
& v_{\alpha}^{+}= \begin{cases}\lim _{\alpha^{\prime} \rightarrow \alpha^{-}} \bigvee_{t \in T}\left(u_{t}\right)_{\alpha^{\prime}}^{+} & \alpha \in \operatorname{Disc}\left(v^{+}\right), \\
\bigvee_{t \in T}\left(u_{t}\right)_{\alpha}^{+} & \text {otherwise },\end{cases}
\end{aligned}
$$

for $\alpha \in(0,1]$, where Disc $\left(v^{+}\right)$is the set of all discontinuous points of $v^{+}$, which is at most countable; while

$$
v_{0}^{-}=\bigwedge_{\lambda>0} \bigvee_{t \in T}\left(u_{t}\right)_{\lambda}^{-}, \quad v_{0}^{+}=\bigvee_{t \in T}\left(u_{t}\right)_{0}^{+} .
$$

We define a nested closed intervals $w=\left\{\left[w_{\alpha}^{-}, w_{\alpha}^{+}\right] \mid \alpha \in I\right\}$, where $w^{-}$and $w^{+}$ are functions defined on $I, w_{\alpha}^{-}=\bigvee_{t \in T}\left(u_{t}\right)_{\alpha}^{-}, w_{\alpha}^{+}=\bigvee_{t \in T}\left(u_{t}\right)_{\alpha}^{+}$for $\alpha \in I$. It can be seen that $w_{\alpha}^{-}$is increasing, $w_{\alpha}^{+}$is decreasing on $I, v_{\alpha}^{-}=w_{\alpha}^{-}$for all $\alpha \in(0,1]$ and $v_{\alpha}^{+}$is the smallest $\mathrm{u}$. s. c. function greater than $w_{\alpha}^{+}$and they may differ only at the discontinuous points of $v_{\alpha}^{+}$, i.e., $w_{\alpha}^{+}=v_{\alpha}^{+}$a.e. on $I$. It follows that $d_{p}(w, v)=0$, where $d_{p}(w, v)$ is defined by the following formula as if $w$ is a fuzzy number:

$$
d_{p}(w, v)=\left(\int_{0}^{1}\left(\max \left\{\left|w_{\alpha}^{-}-v_{\alpha}^{-}\right|,\left|w_{\alpha}^{+}-v_{\alpha}^{+}\right|\right\}\right)^{p} d \alpha\right)^{\frac{1}{p}} .
$$

The above representation of supremum is based on the cut-set functions. There is also a representation of supremum based on the membership functions as follows:

Proposition 2[4] Let $\left\{u_{t} \mid t \in T\right\}$ be a family of fuzzy numbers. If the family is bounded above, $v$ the supremum of the set, then the membership function of $v$ is given by the following formula:

$$
\begin{aligned}
v(x) & = \begin{cases}\bigwedge_{t \in T} l_{u_{t}}(x), & x<v_{1}^{-} ; \\
c l\left(\bigvee u_{u_{t}}(x)\right), & \text { otherwise. }\end{cases} \\
& = \begin{cases}\bigwedge_{t \in T} l_{u_{t}}(x), & x<v_{1}^{-} ; \\
1, & x=v_{1}^{-} \\
\lim _{x^{\prime} \rightarrow x^{-}} \bigvee_{t \in T} u_{t}\left(x^{\prime}\right), & x \in \operatorname{Disc}^{+}(v) ; \\
\bigvee_{t \in T} u_{t}(x), & \text { otherwise. }\end{cases}
\end{aligned}
$$

Where $\operatorname{Disc}^{+}(v)$ is the set of all discontinuous points of $v$ greater than $v_{1}^{-}$, which is at most countable since $v$ is quasiconvex as a real function. The closure is taken in the induced fuzzy topological space $\left(I^{R}, \omega(\tau)\right)$, where $\tau$ is the usual topology on $R$. (For related concepts on fuzzy topology, we refer to [6] and [7].) follows:

Now, we consider the fuzzy set $w^{\prime}$ whose membership function is defined as

$$
w^{\prime}(x)= \begin{cases}\bigwedge_{t \in T} l_{u_{t}}(x) & x \leq v_{1}^{-} \\ \bigvee_{t \in T} r_{u_{t}}(x) & \text { otherwise }\end{cases}
$$


Note that $w^{\prime}\left(v_{1}^{-}\right)=1$ and $v$ is the smallest $\mathrm{u}$. $\mathrm{s}$. c. function greater than $w^{\prime} . w^{\prime}$ is u. s. c. if only if $w^{\prime} \in E^{1}$, i.e., $w^{\prime}=v$. $w^{\prime}$ can also be equivalently defined as follows: $w^{\prime}=\left(l_{w^{\prime}}, r_{w^{\prime}}\right)$, the definitions of $l_{w^{\prime}}$ and $r_{w^{\prime}}$ are similar to the case of fuzzy numbers, that is $l_{w^{\prime}}(x)=\bigwedge_{t \in T} l_{u_{t}}(x), r_{w^{\prime}}(x)=\bigvee_{t \in T} r_{u_{t}}(x)$. By the definition of $v$, we have $l_{w^{\prime}}=l_{v}$ and $r_{w^{\prime}}(x) \neq r_{v}(x)$ only if $x \in \operatorname{Disc}^{+}(v)$. Thus $r_{w^{\prime}}$ and $r_{v}$ differ at most on a countable set.

Now, we proceed to consider the relation between $w$ and $w^{\prime}$. In fact, $w$ does not necessarily correspond to the cut-set function of a fuzzy number, since $w^{+}$may not be left continuous. In general, $w$ may not even be a cut-set function of a fuzzy set. But we can define a fuzzy set $w^{*}$ on $R$ according to $w$ as follows:

$$
w^{*}(x)=\sup \left\{\alpha \in I: x \in\left[w_{\alpha}^{-}, w_{\alpha}^{+}\right]\right\} .
$$

It is obvious that $w^{*}(x)=v(x)$ for $x \in R$. In fact, $v(x)=\sup \left\{\alpha \in I: x \in\left[v_{\alpha}^{-}, v_{\alpha}^{+}\right]\right\}$ and $\left[w_{\alpha}^{-}, w_{\alpha}^{+}\right] \subseteq\left[v_{\alpha}^{-}, v_{\alpha}^{+}\right] \subseteq\left[w_{\alpha-\varepsilon}^{-}, w_{\alpha-\varepsilon}^{+}\right]$for $\alpha \in I$ and arbitrary $\varepsilon>0$ such that $\alpha-\varepsilon \geq 0$. So $w_{\alpha}^{-}=\left(w^{*}\right)_{\alpha}^{-}$for all $\alpha \in(0,1]$ and $w_{\alpha}^{+} \neq\left(w^{*}\right)_{\alpha}^{+}$only if $\alpha \in \operatorname{Disc}\left(v^{+}\right)$. Since $v(x) \neq w^{\prime}(x)$ if only $x \in \operatorname{Disc}^{+}(v)$. So $w^{*}(x) \neq w^{\prime}(x)$ only if $x \in \operatorname{Disc}^{+}(v)$. The following example shows that $w^{+} \neq v^{+}$and $w^{\prime} \neq v$ in general.

Example 1 Let $v=\chi_{[0,1]}+\frac{1}{2} \chi_{[1,2]}+\frac{1}{3} \chi_{[2,3]}$, where $\chi_{A}$ denotes the characteristic function of a set $A \subseteq R$. For $n=3,4, \cdots$, let

$$
u_{n}(x)= \begin{cases}1 & x \in\left[0,1-\frac{1}{n}\right], \\ \frac{1}{2} & x \in\left(1-\frac{1}{n}, 2\right], \\ \frac{1}{3}-\frac{1}{n} & x \in(2,3], \\ 0 & \text { otherwise }\end{cases}
$$

then, clearly, $v=\bigvee_{n=3}^{+\infty} u_{n}$,

$$
\begin{gathered}
w_{\alpha}^{+}=\bigvee_{n=3}^{+}\left(u_{n}\right)_{\alpha}^{+}=\left\{\begin{array}{cc}
3 & \alpha \in\left[0, \frac{1}{3}\right), \\
2 & \alpha \in\left[\frac{1}{3}, \frac{1}{2}\right], \\
1 & \alpha \in\left(\frac{1}{2}, 1\right],
\end{array}\right. \\
v_{\alpha}^{+}=\left\{\begin{array}{l}
\alpha \in\left[0, \frac{1}{3}\right], \\
2 \quad \alpha \in\left(\frac{1}{3}, \frac{1}{2}\right], \\
1 \quad \alpha \in\left(\frac{1}{2}, 1\right],
\end{array}\right.
\end{gathered}
$$

and

$$
w^{\prime}(x)=\left\{\begin{array}{cl}
1 & x \in[0,1), \\
\frac{1}{2} & x \in[1,2], \\
\frac{1}{3} & x \in(2,3] \\
0 & \text { otherwise }
\end{array}\right.
$$

It follows that $w_{\frac{1}{3}}^{+} \neq v_{\frac{1}{3}}^{+}, \frac{1}{3} \in \operatorname{Disc}\left(v^{+}\right)$and $w^{\prime}(1) \neq v(1), 1 \in \operatorname{Disc}^{+}(v)$.

Lemma 1 Assume that $\left\{u_{n}\right\}$ and $v$ are given as in Proposition 1, $w$ is defined as above, and $u \in E^{1}, u \leq v$. Suppose that support $(v) \subseteq[a, b]$ and $a \leq w_{0}^{-}=$ 
$\bigvee_{t \in T}\left(u_{t}\right)_{0}^{-}$. If there exists $n$ such that $\frac{b-a}{n}<\frac{\varepsilon}{2}$,

$$
w_{\frac{i}{n}}^{-}<u_{\frac{i}{n}}^{-}+\frac{\varepsilon}{2}, \quad i=0,1, \ldots, n-1
$$

and

$$
w_{\frac{i}{n}}^{+}<u_{\frac{i}{n}}^{+}+\frac{\varepsilon}{2}, \quad i=1, \ldots, n
$$

then $d_{1}(u, v)<2 \varepsilon$

Proof Since $d_{1}(w, v)=0$, so we only need to show that $d_{1}(u, w)<2 \varepsilon$. First, we show that $\left\|u^{-}-w^{-}\right\|_{1}<\varepsilon$. Define two simple functions $h_{1}$ and $h_{1}^{\prime}$ on $[0,1]$ as follows: $h_{1}(\alpha)=w_{\frac{i}{n}}^{-}, h_{1}^{\prime}(\alpha)=u_{\frac{i}{n}}^{-}$, for $\alpha \in\left[\frac{i}{n}, \frac{i+1}{n}\right), i=0,1, \ldots, n-1 ; h_{1}(1)=w_{1}^{-}, h_{1}^{\prime}(1)=$ $u_{1}^{-}$. Clearly, by (1) and the monotonicity of $w_{\alpha}^{-}$and $u_{\alpha}^{-}$, we have $h_{1}(\alpha) \leq w_{\alpha}^{-}$, $h_{1}^{\prime}(\alpha) \leq u_{\alpha}^{-}$and $0 \leq h_{1}(\alpha)-h_{1}^{\prime}(\alpha)<\frac{\varepsilon}{2}$ for all $\alpha \in[0,1]$. Then $\int_{0}^{1}\left(w_{\alpha}^{-}-h_{1}(\alpha)\right) d \alpha=$ $\sum_{i=0}^{n-1} \int_{\frac{i}{n}}^{\frac{i+1}{n}}\left(w_{\alpha}^{-}-h_{1}(\alpha)\right) d \alpha \leq \sum_{i=0}^{n-1}\left(w_{\frac{i+1}{n}}^{-}-w_{\frac{i}{n}}^{-}\right) \frac{1}{n}=\frac{1}{n}\left(w_{1}^{-}-w_{0}^{-}\right)<\frac{1}{n}(b-a)<\frac{\varepsilon}{2}$.

Thus, we have

$$
\begin{aligned}
\left\|w^{-}-u^{-}\right\|_{1} & =\int_{0}^{1}\left(w_{\alpha}^{-}-h_{1}(\alpha)\right) d \alpha+\int_{0}^{1}\left(h_{1}(\alpha)-u_{\alpha}^{-}\right) d \alpha \\
& \leq \int_{0}^{1}\left(w_{\alpha}^{-}-h_{1}(\alpha)\right) d \alpha+\int_{0}^{1}\left(h_{1}(\alpha)-h_{1}^{\prime}(\alpha)\right) d \alpha \\
& <\frac{\varepsilon}{2}+\frac{\varepsilon}{2}=\varepsilon .
\end{aligned}
$$

Second, we show that $\left\|w^{+}-u^{+}\right\|_{1}<\varepsilon$. Similar to the above case, we also define two simple functions $h_{2}$ and $h_{2}^{\prime}$ on $[0,1]$ as follows: $h_{2}(0)=w_{0}^{+}, h_{2}^{\prime}(0)=u_{0}^{+}$; $h_{2}(\alpha)=w_{\frac{i}{n}}^{+}, h_{2}^{\prime}(\alpha)=w_{\frac{i}{n}}^{+}$, for $\alpha \in\left(\frac{i-1}{n}, \frac{i}{n}\right], i=1,2 \ldots, n$. By a similar argument as above, we have $\int_{0}^{1}\left(w_{\alpha}^{+}-h_{2}(\alpha)\right) d \alpha<\frac{\varepsilon}{2}$ and $\left\|u^{+}-w^{+}\right\|_{1}<\varepsilon$. Thus, $d_{1}(u, w)<2 \varepsilon$. So the proof is completed.

Based on the above discussion, we have the following algorithm of computing the supremum of fuzzy numbers via the $L_{1}$ metric $d_{1}$.

Theorem 1 Under the hypothesis of Proposition 1.

(i) Choose $a, b$ such that $a \leq w_{0}^{-}$and $b>v_{0}^{+}=\bigvee_{t \in T}\left(u_{t}\right)_{0}^{+}$;

(ii) Pick nature number $n$ such that $\frac{b-a}{n}<\frac{\varepsilon}{2}$.

For $i=0,1, \cdots n-1$, choose $t_{i} \in T$ such that

$$
w_{\frac{i}{n}}^{-}<\left(u_{t_{i}}\right)_{\frac{i}{n}}^{-}+\frac{\varepsilon}{2} .
$$

For $j=1,2, \cdots, n$, choose $t_{n+j-1} \in T$ such that

$$
w_{\frac{j}{n}}^{+}<\left(u_{t_{n+j-1}}\right)_{\frac{j}{n}}^{+}+\frac{\varepsilon}{2} .
$$

Let $u=\bigvee_{i=0}^{2 n-1} u_{t_{i}}$, then $d_{1}(u, v)<2 \varepsilon$. 
In the following, we consider the case when the family of fuzzy numbers is given by their membership functions. It can be seen that the calculations of approximation with respect to supremum via $L_{1}$ metric can be carried out in a similar way as in the cut-set case. By the geometric meaning of integration and the integration variable transformation, we have the following lemma.

Lemma 2 For $u, v \in E^{1}$, we have

$$
\left\|u^{-}-v^{-}\right\|_{1}=\int_{\min \left\{u_{0}^{-}, v_{0}^{-}\right\}}^{\max \left\{u_{1}^{-}, v_{1}^{-}\right\}}\left|l_{u}(x)-l_{v}(x)\right| d x
$$

and

$$
\left\|u^{+}-v^{+}\right\|_{1}=\int_{\min \left\{u_{1}^{+}, v_{1}^{+}\right\}}^{\max \left\{u_{0}^{+}, v_{0}^{+}\right\}}\left|r_{u}(x)-r_{v}(x)\right| d x .
$$

Proof Here, we only prove (3). The proof for (4) is similar. In order to prove this lemma, we resort to the Lebesgue measure of real two-dimensional space $R^{2}$. Let

$$
\begin{gathered}
A_{1}=\left\{(x, \alpha) \in R^{2} \mid \min \left\{u_{\alpha}^{-}, v_{\alpha}^{-}\right\} \leq x \leq \max \left\{u_{\alpha}^{-}, v_{\alpha}^{-}\right\} \text {and } 0 \leq \alpha \leq 1\right\}, \\
A_{2}=\left\{(x, \alpha) \in R^{2} \mid u_{\alpha}^{-} \leq x<\lim _{\alpha^{\prime} \rightarrow \alpha^{+}} u_{\alpha^{\prime}}^{-} \text {and } \alpha \in \operatorname{Disc}\left(u^{-}\right)\right\}, \\
A_{3}=\left\{(x, \alpha) \in R^{2} \mid v_{\alpha}^{-} \leq x<\lim _{\alpha^{\prime} \rightarrow \alpha^{+}} v_{\alpha^{\prime}}^{-} \text {and } \alpha \in \operatorname{Disc}\left(v^{-}\right)\right\}, \\
A=A_{1} \bigcup A_{2} \bigcup A_{3}
\end{gathered}
$$

and

$$
\begin{aligned}
B=\left\{(x, \alpha) \in R^{2} \mid \quad\right. & \min \left\{l_{u}(x), l_{v}(x)\right\} \leq \alpha \leq \max \left\{l_{u}(x), l_{v}(x)\right\} \text { and } \\
& \left.\min \left\{u_{0}^{-}, v_{0}^{-}\right\} \leq x \leq \max \left\{u_{1}^{-}, v_{1}^{-}\right\}\right\}
\end{aligned}
$$

Here $\operatorname{Disc}\left(u^{-}\right)$and $\operatorname{Disc}\left(v^{-}\right)$are the sets of all discontinuous points of $u^{-}$ and $v^{-}$respectively. Clearly, $A_{i}(i=1,2,3)$ and $B$ are measurable. The Lebesgue measure of $A_{i}(i=1,2,3), A$ and $B$ are denoted by $m\left(A_{i}\right)(i=1,2,3), m(A)$ and $m(B)$, respectively. By the meaning of integration and Lebesgue measure of $R^{2}$, we have $m\left(A_{1}\right)=\left\|u^{-}-v^{-}\right\|_{1}$ and $m(B)=\int_{\min \left\{u_{0}^{-}, v_{0}^{-}\right\}}^{\max \left\{u_{-}^{-}, v^{-}\right\}}\left|l_{u}(x)-l_{v}(x)\right| d x$.

Now, we show that $A=B$. First, for each $(x, \alpha) \in A$, there are three cases: Case 1. $(x, \alpha) \in A_{1}$, then $\min \left\{u_{\alpha}^{-}, v_{\alpha}^{-}\right\} \leq x \leq \max \left\{u_{\alpha}^{-}, v_{\alpha}^{-}\right\}$, so $\min \left\{u_{0}^{-}, v_{0}^{-}\right\} \leq$ $x \leq \max \left\{u_{1}^{-}, v_{1}^{-}\right\}$. Without loss of generality, we can suppose that $u_{\alpha}^{-} \leq v_{\alpha}^{-}$, then $u_{\alpha}^{-} \leq x \leq v_{\alpha}^{-}$, thus $u_{\alpha}^{-} \leq x<u_{1}^{-}$or $x \geq u_{1}^{-}$and $l_{v}(x)=v(x) \leq \alpha$. So $l_{u}(x)=u(x) \geq \alpha$ when $u_{\alpha}^{-} \leq x \leq u_{1}^{-}$and $l_{u}(x)=1$ when $x \geq u_{1}^{-}$. Thus, $l_{v}(x) \leq \alpha \leq l_{u}(x)$, i.e., $\min \left\{l_{u}(x), l_{v}(x)\right\} \leq \alpha \leq \max \left\{l_{u}(x), l_{v}(x)\right\}$. So $(x, \alpha) \in B$.

Case 2. $(x, \alpha) \in A_{2}$. Since $u_{\alpha}^{-} \leq x<\lim _{\alpha^{\prime} \rightarrow \alpha^{+}} u_{\alpha^{\prime}}^{-}$and $u_{\alpha}^{-}$is increasing, so $u_{0}^{-} \leq x \leq u_{1}^{-}$. As $l_{u}(x)$ is upper semicontinuous on $R, l_{u}(x)=\alpha$. Thus, $(x, \alpha) \in B$. Case 3. $(x, \alpha) \in A_{3}$. Similarly to the case 2 , we can show that $(x, \alpha) \in B$.

Thus, by the above discussion we have $A \subseteq B$. 
Conversely, for each $(x, \alpha) \in B$, we will show that if $(x, \alpha) \notin A_{2} \bigcup A_{3}$, then $(x, \alpha) \in A_{1}$. Since $(x, \alpha) \in B, \min \left\{l_{u}(x), l_{v}(x)\right\} \leq \alpha \leq \max \left\{l_{u}(x), l_{v}(x)\right\}$. Since $0 \leq \alpha \leq 1$, we have $\min \left\{u_{\alpha}^{-}, v_{\alpha}^{-}\right\} \leq x \leq \max \left\{u_{\alpha}^{-}, v_{\alpha}^{-}\right\}$. In fact, if $x<$ $\min \left\{u_{\alpha}^{-}, v_{\alpha}^{-}\right\}$, then $l_{u}(x)=u(x)<\alpha$ and $l_{v}(x)=v(x)<\alpha$ which contradict $\alpha \leq \max \left\{l_{u}(x), l_{v}(x)\right\}$; if $x>\max \left\{u_{\alpha}^{-}, v_{\alpha}^{-}\right\}$, since $x \leq \max \left\{u_{1}^{-}, v_{1}^{-}\right\}$, so $0 \leq \alpha<1$. As $(x, \alpha) \notin A_{2} \bigcup A_{3}$, i.e., $\alpha \notin \operatorname{Disc}\left(u^{-}\right) \bigcup \operatorname{Disc}\left(v^{-}\right)$, so $l_{u}(x)>\alpha$ and $l_{v}(x)>\alpha$ which contradict $\alpha>\min \left\{l_{u}(x), l_{v}(x)\right\}$. Hence, for each $(x, \alpha) \in B,(x, \alpha) \in A$, i.e., $B \subseteq A$.

Thus, $A=B$. Since $\operatorname{Disc}\left(u^{-}\right) \cup \operatorname{Disc}\left(v^{-}\right)$is at most countable, hence $m\left(A_{2} \cup A_{3}\right)=0$, thus $m\left(A_{1}\right)=m(B)$. The proof of (3) is thus completed.

Remark 2 From Lemma 2, it can be seen that the integral defined via cut-set can be represented by the integral defined via corresponding membership functions which is more direct in certain cases. By the properties of $l_{u}, l_{v}$ and $r_{u}, r_{v}$, we can arbitrarily extend the integration interval of the formulas on the right hand side of (3) and (4), but their integration values remain the same:

$$
\int_{-\infty}^{+\infty}\left|l_{u}(x)-l_{v}(x)\right| d x=\int_{\min \left\{u_{0}^{-}, v_{0}^{-}\right\}}^{\max \left\{u_{1}^{-}, v_{1}^{-}\right\}}\left|l_{u}(x)-l_{v}(x)\right| d x
$$

and

$$
\int_{-\infty}^{+\infty}\left|r_{u}(x)-r_{v}(x)\right| d x=\int_{\min \left\{u_{1}^{+}, v_{1}^{+}\right\}}^{\max \left\{u_{0}^{+}, v_{0}^{+}\right\}}\left|r_{u}(x)-r_{v}(x)\right| d x
$$

Now, we define another metric on $E^{1}$, which is based on the membership functions of fuzzy numbers. For any $u, v \in E^{1}$, define

$$
d_{1}^{*}(u, v)=\int_{-\infty}^{+\infty}\left(\left|l_{u}(x)-l_{v}(x)\right|+\left|r_{u}(x)-r_{v}(x)\right|\right) d x .
$$

Clearly, $d_{1}^{*}$ is a metric on $E^{1}$.

By the definition of $d_{1}$, we have

$$
\frac{1}{2} \int_{0}^{1}\left(\left|u_{\alpha}^{-}-v_{\alpha}^{-}\right|+\left|u_{\alpha}^{+}-v_{\alpha}^{+}\right|\right) d \alpha \leq d_{1}(u, v) \leq \int_{0}^{1}\left(\left|u_{\alpha}^{-}-v_{\alpha}^{-}\right|+\left|u_{\alpha}^{+}-v_{\alpha}^{+}\right|\right) d \alpha .
$$

So by Lemma 2, Remark 2 and the definition of $d_{1}^{*}$, we have the following theorem.

Theorem 2 Let $u, v \in E^{1}$, then

$$
\frac{1}{2} d_{1}^{*}(u, v) \leq d_{1}(u, v) \leq d_{1}^{*}(u, v) .
$$


Remark 3 The metric $d_{1}^{*}$ on $E^{1}$ is based on the membership functions of fuzzy numbers. It is more direct than the $L_{1}$ metric $d_{1}$ in certain cases since $d_{1}$ is based on the cut-set functions. From Theorem 2, we can see that the metrics $d_{1}$ and $d_{1}^{*}$ are uniformly equivalent. So they have the same topological properties. Hence, the metric $d_{1}^{*}$ is also approximate to the supremum and it is computable.

Lemma 3 Assume that $\left\{u_{n}\right\}$ and $v$ are given as in Proposition 1, $w$ is defined as above, and $u \in E^{1}, u \leq v$. Let $a=u_{0}^{-}$and $b=v_{0}^{+}$. Suppose that there exists natural number $n$ such that $\frac{b-a}{n}<\frac{\varepsilon}{2}$,

$$
l_{u}\left(x_{i}\right)<l_{w^{\prime}}\left(x_{i}\right)+\frac{\varepsilon}{2(b-a)}, \quad i=1,2, \cdots, n,
$$

and

$$
r_{w^{\prime}}\left(x_{i}\right)<r_{u}\left(x_{i}\right)+\frac{\varepsilon}{2(b-a)}, \quad i=1,2, \cdots, n,
$$

where $x_{i}=a+\frac{i(b-a)}{n}$. Then $d_{1}(u, v)<2 \varepsilon$.

Proof By Lemma 2 and Remark 2, we have

$$
d_{1}(u, v) \leq \int_{a}^{b}\left(l_{u}(x)-l_{v}(x)\right) d x+\int_{a}^{b}\left(r_{v}(x)-r_{u}(x)\right) d x .
$$

Since $l_{v}=l_{w^{\prime}}$ on $[a, b]$ and $r_{v}=r_{w^{\prime}}$ a.e. on $[a, b]$, so

$$
d_{1}(u, v) \leq \int_{a}^{b}\left(l_{u}(x)-l_{w^{\prime}}(x)\right) d x+\int_{a}^{b}\left(r_{w^{\prime}}(x)-r_{u}(x)\right) d x .
$$

First, we show that $\int_{a}^{b}\left(l_{u}(x)-l_{w^{\prime}}(x)\right) d x<\varepsilon$. Define two simple functions $h_{1}, h_{1}^{\prime}$ on $[a, b]$ as follows: $h_{1}\left(x_{0}\right)=l_{w^{\prime}}\left(x_{0}\right), h_{2}\left(x_{0}\right)=l_{u}\left(x_{0}\right) ; h_{1}(x)=l_{w^{\prime}}\left(x_{i}\right), h_{1}^{\prime}(x)=$ $l_{u}\left(x_{i}\right)$, for $x \in\left(x_{i-1}, x_{i}\right], i=1,2, \cdots, n$. By $(5), h_{1}^{\prime}(x)-h_{1}(x)<\frac{\varepsilon}{2(b-a)}$ for $x \in[a, b]$. And $\int_{a}^{b}\left(h_{1}(x)-l_{w^{\prime}}(x)\right) d x=\sum_{i=1}^{n} \int_{x_{i-1}}^{x_{i}}\left(h_{1}(x)-l_{w^{\prime}}(x)\right) d x \leq \sum_{i=1}^{n} \int_{x_{i-1}}^{x_{i}}\left(l_{w^{\prime}}\left(x_{i}\right)-\right.$ $\left.l_{w^{\prime}}\left(x_{i-1}\right)\right) d x=\left(l_{w}^{\prime}\left(x_{n}\right)-l_{w^{\prime}}\left(x_{0}\right)\right) \frac{b-a}{n}<\frac{\varepsilon}{2}$. So

$$
\begin{aligned}
\int_{a}^{b}\left(l_{u}(x)-l_{w^{\prime}}(x)\right) d x & \leq \int_{a}^{b}\left(h_{1}^{\prime}(x)-l_{w^{\prime}}(x)\right) d x \\
& =\int_{a}^{b}\left(h_{1}^{\prime}(x)-h_{1}(x)\right) d x+\int_{a}^{b}\left(h_{1}(x)-l_{w^{\prime}}(x) d x\right. \\
& <\frac{\varepsilon}{2}+\frac{\varepsilon}{2}=\varepsilon .
\end{aligned}
$$

Similarly, $\int_{a}^{b}\left(r_{w^{\prime}}(x)-r_{u}(x)\right) d x<\varepsilon$. The proof is thus completed.

By the discussion of Lemma 2, we have the following algorithm on the supremum of fuzzy numbers based on membership functions.

Theorem 3 Assume that $\left\{u_{t} \mid t \in T\right\}$ and $v$ are given as in Proposition 1, $w$ is defined as above. 
(i) Choose $b>v_{0}^{+}=\bigvee_{t \in T}\left(u_{t}\right)_{0}^{+}$, pick $t^{\prime} \in T$ and let $\left(u_{t^{\prime}}\right)_{0}^{-}=a$;

(ii) Choose a natural number $n$ such that $\frac{b-a}{n}<\frac{\varepsilon}{2}$.

For $i=1,2, \cdots, n$, choose $t_{i} \in T$ such that

$$
r_{w^{\prime}}\left(a+\frac{i(b-a)}{n}\right)<r_{u_{t_{i}}}\left(a+\frac{i(b-a)}{n}\right)+\frac{\varepsilon}{2(b-a)} .
$$

For $j=1,2, \cdots, n$, choose $t_{n+j} \in T$ such that

$$
l_{u_{t_{n+j}}}\left(a+\frac{j(b-a)}{n}\right)<l_{w^{\prime}}\left(a+\frac{j(b-a)}{n}\right)+\frac{\varepsilon}{2(b-a)} .
$$

Let $u_{t^{\prime}}=u_{t_{0}}, u=\vee_{i=0}^{2 n} u_{t_{i}}$, then $d_{1}(u, v)<2 \varepsilon$.

Remark 4 Note that in the proof of Lemma 3, we only used the values of $u$ at the isolated points:

$$
x_{i}=a+\frac{i(b-a)}{n}, \quad i=1,2, \cdots, n .
$$

Thus, if we define $u^{\prime}=\left(l_{u^{\prime}}, r_{u^{\prime}}\right)$ such that $l_{u^{\prime}}\left(x_{i}\right)=l_{u}\left(x_{i}\right), r_{u^{\prime}}\left(x_{i}\right)=r_{u}\left(x_{i}\right)$, $i=1,2, \cdots, n$, and let $u^{\prime}$ be linear between $x_{i}$ and $x_{i+1}, i=1,2, \cdots, n-1$, then we also have $d_{1}\left(u^{\prime}, v\right)<2 \varepsilon$. Note that $l_{u^{\prime}}\left(x_{i}\right)=l_{u_{t_{i}}}\left(x_{i}\right), r_{u^{\prime}}\left(x_{i}\right)=l_{u_{t_{i+n}}}\left(x_{i}\right)$ for some $t_{i}, t_{i+n} \in T, i=1,2, \cdots, n$, and $u^{\prime}$ is piecewise linear and hence continuous, thus we have the following:

Theorem 4 Under the hypothesis of Proposition 2, for every $\epsilon>0$ there is a piecewise linear fuzzy number $u^{\prime}$ which is determined by a finite number of values of a finite number of fuzzy numbers of $\left\{u_{t} \mid t \in T\right\}$ such that $d_{1}\left(u^{\prime}, v\right)<2 \varepsilon$.

\section{CALCULATIONS VIA $L_{p}(p \geq 1)$ METRICS}

In this section, we discuss the finite approximate algorithm for supremum with respect to the general $L_{p}$ metrics. The situation will be much more complicated than the case when $p=1$.

Definition 1 Let $f$ be a monotone left continuous function from $[0,1]$ to $R$. Suppose that $a \leq f(x) \leq b$ for all $x \in[0,1]$. For each $\varepsilon>0$, pick natural number $n$ such that $\frac{b-a}{n}<\frac{\varepsilon}{2}$. Let $E_{i}=\left\{x \in[0,1] \mid a+\frac{i}{n}(b-a) \leq f(x) \leq a+\frac{i+1}{n}(b-a)\right\}$, $i=0,1, \cdots, n-1 ; E_{j}^{\prime}=\left\{x \in[0,1] \mid f(x)=a+\frac{j}{n}(b-a)\right\}, j=0,1, \cdots, n$. Now we define the partition points $x_{j}$ as follows:

Case 1. $E_{j}^{\prime}$ is an interval (the interval is right closed since $f$ is left continuous), take $x_{j}=\sup E_{j}^{\prime}$.

Case 2. $E_{j}^{\prime}$ is an empty set. If $f$ is increasing, take $x_{j}=\sup \left\{\bigcup_{i \leq j} E_{i}\right\}$; if $f$ is decreasing, take $x_{j}=\inf \left\{\bigcup_{i \leq j} E_{i}\right\}$. 
Clearly, if $f$ is increasing, then $0=x_{0} \leq x_{1} \leq \cdots \leq x_{n}=1$; if $f$ is decreasing, then $1=x_{0} \geq x_{1} \geq \cdots \geq x_{n}=0$. So they form a set of partitioning points for $[0,1]$. It may happen that $\left\{x_{i} \mid i=0,1, \cdots n\right\}$ have repeated points. For our need we can rename these partition points and make them have no repeated points. So $0=x_{0}<x_{1}<\cdots<x_{n}=1$ when $f$ is increasing and $1=x_{0}>x_{1}>\cdots>x_{n}=0$ when $f$ is decreasing.

Remark 5 Based on the above partition and by the monotonicity of the function $f$, we have $0 \leq f\left(x_{i+1}\right)-f(x)<\frac{\varepsilon}{2}$ for $x \in\left(x_{i}, x_{i+1}\right)$ when $f$ is increasing and $0 \leq f(x)-f\left(x_{i}\right)<\frac{\varepsilon}{2}$ for $x \in\left(x_{i+1}, x_{i}\right)$ when $f$ is decreasing.

Lemma 4 Assume that $w$ and and $v$ are given as in Proposition 1, $u$ is a fuzzy number such that $u \leq v$. Let $u_{0}^{-}=a, w_{1}^{-}<b$. For the two functions $w^{+}$and $w^{-}$, pick partitioning points $1=x_{0}>x_{1}>\cdots>x_{n}=0$ and $0=y_{0}<y_{1}<\cdots<$ $y_{m}=1$ of $[0,1]$ respectively defined in Definition 1. If the following conditions are satisfied, then $d_{p}(u, v)<\left(1+2^{\frac{1}{p}}\right) \varepsilon$.

(i) $w_{x_{i}}^{+}<u_{x_{i}}^{+}+\frac{\varepsilon}{2}, \quad i=0,1, \cdots, n-1$.

(ii) For every $y_{j}, j=0,1, \cdots, m-1$, there exists $y_{j}^{\prime} \in\left(y_{j}, y_{j+1}\right), y_{j}^{\prime}-y_{j}<\frac{1}{m}\left(\frac{\varepsilon}{b-a}\right)^{p}$ and $w_{y_{j}^{\prime}}^{-}<u_{y_{j}^{\prime}}^{-}+\frac{\varepsilon}{2}$.

Proof First, we prove that $\left\|v^{+}-w^{+}\right\|_{p}<\varepsilon$. For each $x \in[0,1] \backslash\left\{x_{i} \mid i=1, \cdots, n\right\}$, there exists a partitioning interval $\left(x_{i+1}, x_{i}\right)$ such that $x \in\left(x_{i+1}, x_{i}\right)$. Since $w^{+}$ is decreasing on $[0,1]$, so $\lim _{x \rightarrow x_{i+1}^{+}} w_{x}^{+}$exists, and it is denoted by $w_{x_{i+1}^{+}}^{+}$. So $w_{x}^{+} \leq w_{x_{i+1}^{+}}^{+}$. By the left continuity of $w^{+}$, we have $0 \leq w_{x_{i+1}^{+}}^{+}-w_{x_{i}}^{+}<\frac{\varepsilon}{2}$. Hence, by (i), $w_{x}^{+}-u_{x}^{+} \leq\left(w_{x_{i+1}^{+}}^{+}-w_{x_{i}}^{+}\right)+\left(w_{x_{i}}^{+}-u_{x_{i}}^{+}\right)<\frac{\varepsilon}{2}+\frac{\varepsilon}{2}=\varepsilon$. Thus for each $x \in[0,1]$, $0 \leq w_{x}^{+}-u_{x}^{+}<\varepsilon$. Since $w_{x}^{+} \neq v_{x}^{+}$only if $x \in \operatorname{disc}\left(v^{+}\right)$which is at most countable. So we have $0 \leq v_{x}^{+}-u_{x}^{+}<\varepsilon$ a.e. on $[0,1]$. Thus $\left\|v^{+}-u^{+}\right\|_{p}<\varepsilon$.

Second, we prove that $\left\|u^{-}-w^{-}\right\|_{p}<2^{\frac{1}{p}} \varepsilon$. For each $y \in\left[y_{j}^{\prime}, y_{j+1}\right]$, by left continuity of $w^{-}$, we have $0 \leq w_{y_{j+1}}^{-}-w_{y_{j}^{\prime}}^{-}<\frac{\varepsilon}{2}$. So $w_{y}^{-}-u_{y}^{-} \leq\left(w_{y_{j+1}}^{-}-w_{y_{j}^{\prime}}^{-}\right)+$ $\left(w_{y_{j}^{\prime}}^{-}-u_{y_{j}^{\prime}}^{-}\right)<\frac{\varepsilon}{2}+\frac{\varepsilon}{2}=\varepsilon$. And for each $y \in\left[y_{j}, y_{j}^{\prime}\right], w_{y}^{-}-u_{y}^{-} \leq w_{1}^{-}-u_{0}^{-}<b-a$. So we have

$$
\begin{aligned}
\left\|u^{-}-w^{-}\right\|_{p} & =\left(\sum_{j=0}^{m-1} \int_{y_{j}}^{y_{j}^{\prime}}\left(w_{y}^{-}-u_{y}^{-}\right)^{p} d y+\sum_{j=0}^{m-1} \int_{y_{j}^{\prime}}^{y_{j}}\left(w_{y}^{-}-u_{y}^{-}\right)^{p} d y\right)^{\frac{1}{p}} \\
& <\left(m(b-a)^{p} \cdot \frac{1}{m}\left(\frac{\varepsilon}{b-a}\right)^{p}+\varepsilon^{p}\right)^{\frac{1}{p}} \\
& =2^{\frac{1}{p}} \varepsilon .
\end{aligned}
$$

So $\left\|u^{-}-v^{-}\right\|_{p}=\left\|u^{-}-w^{-}\right\|_{p}<2^{\frac{1}{p}} \varepsilon$. Thus $d_{p}(u, v)<\left(1+2^{\frac{1}{p}}\right) \varepsilon$. The proof is completed.

Based on the above discussion, we have the following algorithm on the supremum of fuzzy numbers. 
Theorem 5 Assume that $\left\{u_{t} \mid t \in T\right\}$ and $v$ are given as in Proposition 1, $w$ is defined as above.

(i) Choose $b>v_{1}^{-}$, pick $t^{\prime} \in T$, let $\left(u_{t^{\prime}}\right)_{0}^{-}=a$.

(ii) For $w^{+}$, pick its partition points $1=x_{0}>x_{1}>\cdots>x_{n}=0$ defined as Definition 1. For $i=0,1, \cdots, n-1$, choose $t_{i} \in T$ such that

$$
w_{x_{i}}^{+}<\left(u_{t_{i}}\right)_{x_{i}}^{+}+\frac{\varepsilon}{2} .
$$

(iii) For $w^{-}$, pick its partition points $0=y_{0}<y_{1}<\cdots<y_{m}=1$ defined as Definition 1. Let $y_{j}^{\prime}=y_{j}+\frac{1}{m}\left(\frac{\varepsilon}{b-a}\right)^{p} \wedge \wedge\left\{y_{j+1}-y_{j} \mid j=0,1, \cdots, m-1\right\}, j=$ $0,1, \cdots, m-1$. For each $j=0,1, \cdots, m-1$, choose $t_{n+j} \in T$ such that

$$
\begin{gathered}
w_{y_{j}^{\prime}}^{-}<\left(u_{t_{n+j}}\right)_{y_{j}^{\prime}}^{-}+\frac{\varepsilon}{2} . \\
\text { Let } t_{n+m}=t^{\prime} \text { and } u=\bigvee_{i=0}^{n+m} u_{t_{i}} \text {, then } d_{p}(u, v)<\left(1+2^{\frac{1}{p}}\right) \varepsilon .
\end{gathered}
$$

Remark 6 Similar to Theorem 4, we can find a fuzzy number $u^{\prime} \in E^{1}$ whose cutset functions are both linear and determined by a finite number of cut-set values of a finite number of fuzzy numbers of $\left\{u_{t} \mid t \in T\right\}$.

Remark 7 All results in this paper are dually true for infimum.

In this paper, we have shown that the supremum of a family of fuzzy numbers can be finitely approximated via $L_{p}$ metrics and give out the concrete approach to approximate the supremum. As our computation method is finite, it might be executed by computers. As a byproduct, it is proved that the $L_{1}$ metric $d_{1}$ defined via cut-set is equivalent to a metric which can be calculated directly via membership functions. The results in this paper also show that $L_{p}$ metrics are useful metrics on fuzzy number spaces. Because the approximation to the supremum via $L_{p}$ metrics are feasible and computable, moreover, the $L_{p}$ metrics are analytic in nature, so our result may have applications in fuzzy analysis. For example, it could provide a method for the computation of various fuzzy-number-valued integrals.

Acknowledgement. The authors wishes to thank the referee for his kind suggestions which significantly improve the presentation of this paper.

\section{REFERENCES}

1. P. Diamond, P. Kloeden, Metric spaces of fuzzy sets, World Scientific, Singapore, 1994. 
2. T. -H. FAN, "Calculations on the supremum of fuzzy numbers", Proceedings of the north American fuzzy information processing society, 2004, 584-586.

3. T. -H. FAN, "Equivalence of weak convergence and endograph metric convergence for fuzzy number spaces", Fuzzy logic, soft computing and computational intelligence, 11th international Fuzzy Systems Association World Congress, Beijing, 2005, 41-49.

4. T. -H FAN, G. -J WANG, "Endographic approach on supremum and infimum of fuzzy numbers", Information sciences, 159(2004), 221-231.

5. H. HuAng, C. -X. Wu, "Characterizations of $\Gamma$-convergence on fuzzy number spaces", Fuzzy logic, soft computing and computational intelligence, 11th international Fuzzy Systems Association World Congress, Beijing, 2005, 66-70.

6. Y. -M. Liu, M. -K. Luo, Fuzzy topology, World Scientific, Singapore, 1997.

7. G. -J WAng, Theory of L-fuzzy topological spaces, Shaanxi Normal University Press, Xi'an, 1988(In Chinese).

8. C. -X. Wu, C. Wu, "The supremum and infimum of the set of fuzzy numbers and its applications", Journal of mathematical analysis and applications, 210(1997), 499-511.

DAN Wen: Wenling School, Wenling, Zhejiang, 317500, China.

E-mail:wendan0825@163.com.

TAIHe FAn: Department of Mathematics, Zhejiang Sci-Tech University, Hangzhou, Zhejiang, 310018, China.

E-mail: taihefan@hotmail.com. 